\title{
Investigation on a haptic display system for medical ultrasonic elasticity imaging
}

\author{
K. Hamamoto ${ }^{1}$, M. Nakanishi ${ }^{1} \&$ T. Shiina ${ }^{2}$ \\ ${ }^{I}$ Department of Information Media Technology, School of IT and Elec., \\ Tokai University, Japan \\ ${ }^{2}$ Graduate School of Systems and Information Engineering, \\ University of Tsukuba, Japan
}

\begin{abstract}
In the field of medical ultrasonics, ultrasonic elasticity imaging has been developed and used in practical diagnosis. However, unfortunately, elasticity information is now displayed on ordinary 2D screens (for example, CRT). Since elasticity information is haptic information, not visible information, the information is expected to be displayed in "the sense of haptic". In this research, it is attempted to display this elasticity information on a haptic display. In this system, a target tissue is expressed by many small cubes in $3 \mathrm{D}$ virtual space. Each cube has its own elasticity information. When a cube is pressed, not only the cube but some cubes around it are simultaneously moved in the direction of the pressure, but if the pressure disappears, the cubes come back to their original position. This movement of cubes can express a dent in the surface of the body. At that time we can feel haptic sense from the pressed point, which is caused by the mass and elasticity of some cubes around the pressed point. This newlydevised system enables to put the haptic sense to close "Human Haptic". The system also enables one to display the haptic sense in real time after getting elastic information of tissues. This system can realize a virtual palpation system and may realize a tele-palpation system.
\end{abstract}

Keywords: tissue characterization, ultrasonic imaging, elasticity imaging, virtual reality, haptic interface, virtual palpation, real time system. 


\section{Introduction}

Ultrasonic imaging is a widely applied and indispensable technique in medical field currently. Although it has many advantages compared with X-ray imaging techniques, an important weak point is that an ultrasonic echo image is not quantitative. Therefore, there are many research works about quantitative image construction, which is called "Tissue Characterization".

Ultrasonic elasticity imaging is recently one of the most important tissue characterization researches. HITACHI Medical Corporation has already produced the technique on a commercial basis and the equipment is mainly used for detection of tumors of breast cancer [1]. The newest equipment enables one to display the elasticity image in real time. As the technology advances, medical doctors and ultrasonographers are expecting not only a displayed image but "the touch sense" of elasticity information. That is to say, they expect a virtual palpation system.

In recent years, the virtual reality technique has been applied to the medical field, especially the virtual surgery simulator $[2,3]$. Unfortunately, however, the appropriate expression of haptic sense is very difficult and has not been realized yet though the expression is very important. There are two reasons for this. One is the difficulty of measurement of tissue's own elasticity. The mentioned surgery system uses the statistical value, which is not the patient's own data. The other is that computational cost is very high for calculation of the reaction force and movement of the virtual object. It is necessary for a virtual medical system to realize the similar sense as real sense, which consists of not only haptic sense but visual sense. The visual effect is very important to augment haptic sense visually. To realize them is sometimes called "Human Haptics". Human Haptics means putting virtual haptic sense and virtual visual sense close to real haptic sense and real visual sense. Although the Finite Element Method is often used for rendering of visual information and haptic information [4], the computational cost is significantly high.

In this research, the virtual reality technique is applied to medical ultrasonic elasticity imaging. The elasticity information is displayed on a "virtual haptic display". Furthermore, to put the virtual sense close to Human Haptics, new rendering techniques for haptic and visual information are proposed. The system also enables one to display both movements of virtual objects, which correspond to a dent of the surface of the body, and the haptic sense in real time after getting elastic information of tissues. This system will be able to realize a virtual palpation system and may realize a tele-palpation system in the future.

\section{Methods}

To present human haptics for a virtual object, rendering techniques on figure deformation of the object (that is, expression of visual information) and force feed-back from the object (calculation of reaction force, that is, expression of haptic information) are needed. Although the Finite Element Method is chiefly used as a rendering technique in general, the computational cost is very high. 
Therefore, the system cannot display human haptics in real time. Since a real time free hand elasticity imaging system has already been realized in a medical ultrasonic field, it is very important to realize a real time virtual palpation system. In this section, new rendering techniques are proposed.

\subsection{Modelling of tissue}

A 3D finite element model enables one to calculate the precise value, for example, reaction force. However, the human sense is not so high resolution. Therefore, we can reduce the precision of virtual sense within the limits of resolution of human sense. In addition, reduction of computational cost takes precedence over the precision beyond the resolution of human sense. On this consideration, a tissue is expressed as a group of small rigid cubes. Each rigid cube has its own mass and elasticity information.

\subsection{Rendering of deformation of tissue}

When a tissue is pressed at a point, the tissue is deformed around the pressed point. Namely, the tissue is dented. In a virtual palpation system, this movement must be presented exactly. In the proposed system, when a cube is pressed, not only the cube but some cubes around the pressed cube are moved to the direction of the pressure simultaneously. And, if the pressure disappears, the cubes come back to the original position. This movement of cubes can express a dent in the surface of the body for virtual palpation. The reaction force in the event of the return of the cube to its original position is calculated based on mass and elasticity information of the cubes by using a Spring-damper model. The depth of dent for the cubes is determined by the physician or medical doctor based on the mass of cubes subjectively and empirically.

\subsection{Rendering of haptic sense (force feed-back)}

In general, the reaction force, which causes haptic sense, is calculated from the distribution of displacement which is calculated from the distribution of pressure stress by using the Finite Element Method. In this research, however, as mentioned in the previous section, simplification of the calculation of haptic sense is attempted for a real-time system

The sense of stiffness of a virtual object (a pressed cube) is expressed based on the reaction force to the force which is needed to move not only the pressed cube but some cubes around the pressed cube. The parameters are mass and elasticity of the cubes. In this system, we can feel the difference of the sense of stiffness caused by the difference of the depth of tumor, by changing the "weight coefficient" on mass and elasticity for each cube suitably. The coefficients are determined by the physician or medical doctor subjectively and empirically. 


\subsection{Determination of user's own Human Haptics}

These determinations of some coefficients for calculation of the visual effect and the haptic sense are accomplished by a user, based on the user's own sense in advance to practical use. This corresponds to "calibration of Human Haptics". The calibration data is saved in the system. Therefore, the user's own Human haptics can be calculated and presented in real time for practical use by using the user's own calibration file.

\section{Experiment}

\subsection{Haptic device and development environment}

This system uses PHANToM Desktop ${ }^{\mathrm{TM}}$ (SensAble Technologies Inc.) shown in Figure 1 as haptic device. OpenGL is used as the graphic library for construction of virtual space in the visual sense, and GHOST SDK is used as a software library to control PHANToM Desktop ${ }^{\mathrm{TM}}$ and to realize force feed-back, haptic sense.

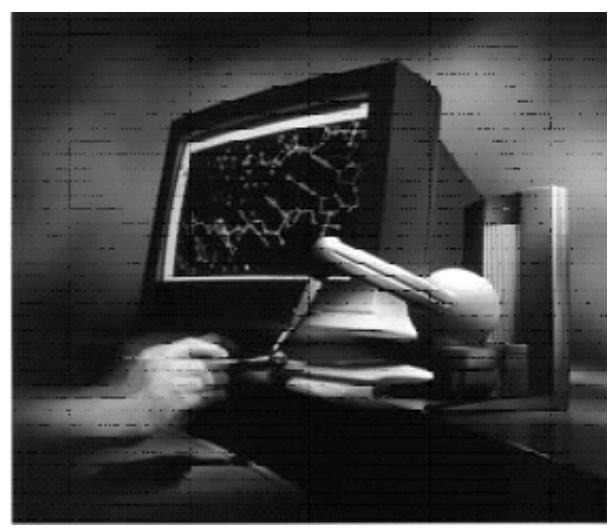

Figure 1: $\quad$ PHANToM Desktop ${ }^{\mathrm{TM}}$.

\subsection{Ultrasonic elasticity imaging}

In this experiment, a result of a phantom experiment of ultrasonic elasticity imaging is used. The phantom form is a cube $(80 \mathrm{~mm} * 80 \mathrm{~mm} * 60 \mathrm{~mm})$. The phantom includes a sphere whose elasticity is different from the surrounding one. The diameter is $15 \mathrm{~mm}$. The elasticity distribution is obtained by a method shown in [1]. The phantom form and the obtained distribution are shown in Figure 2 . 

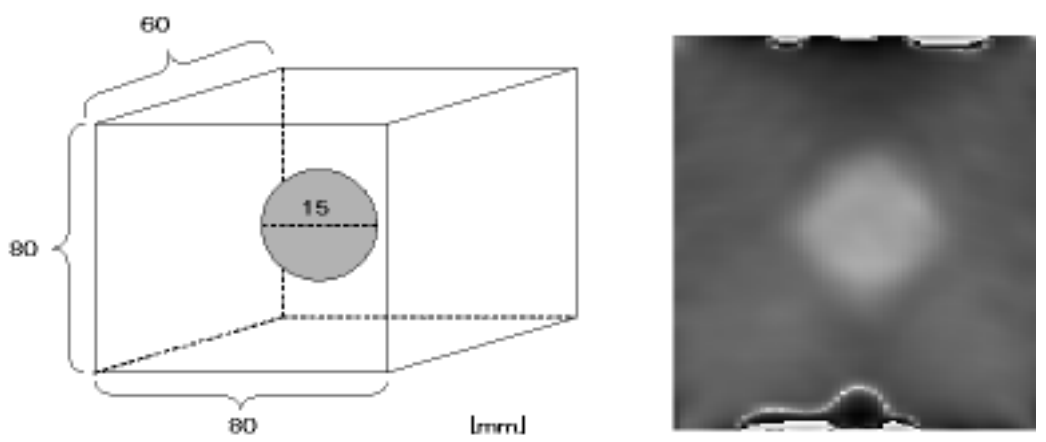

Figure 2: $\quad$ The phantom form (left) and experimental result of elasticity imaging (right).

\subsection{Modelling}

Tissue is constructed in virtual space by many small rigid cubes as shown in Figure 3. This form corresponds to the phantom which is used in section 3.2.

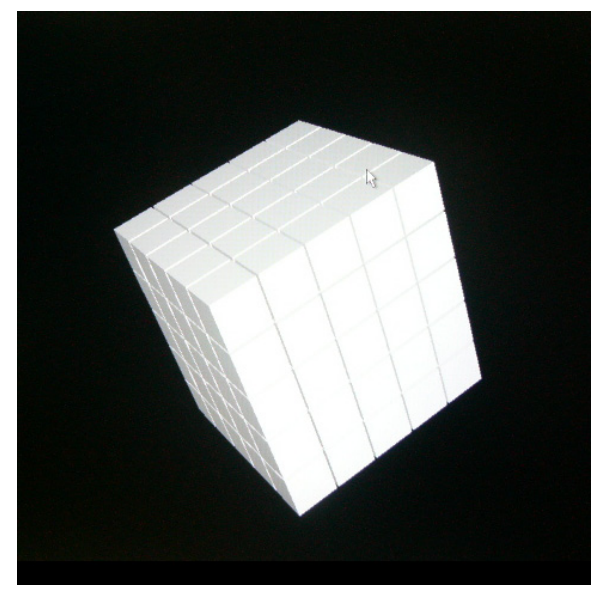

Figure 3: $\quad$ Modelling of tissue.

\subsection{System construction}

The obtained elasticity information of the phantom is applied to the tissue model shown in Figure 3. The 3D tissue elasticity model and PHANToM Desktop ${ }^{\mathrm{TM}}$ are combined into the "Virtual palpation system" shown in Figure 4. 

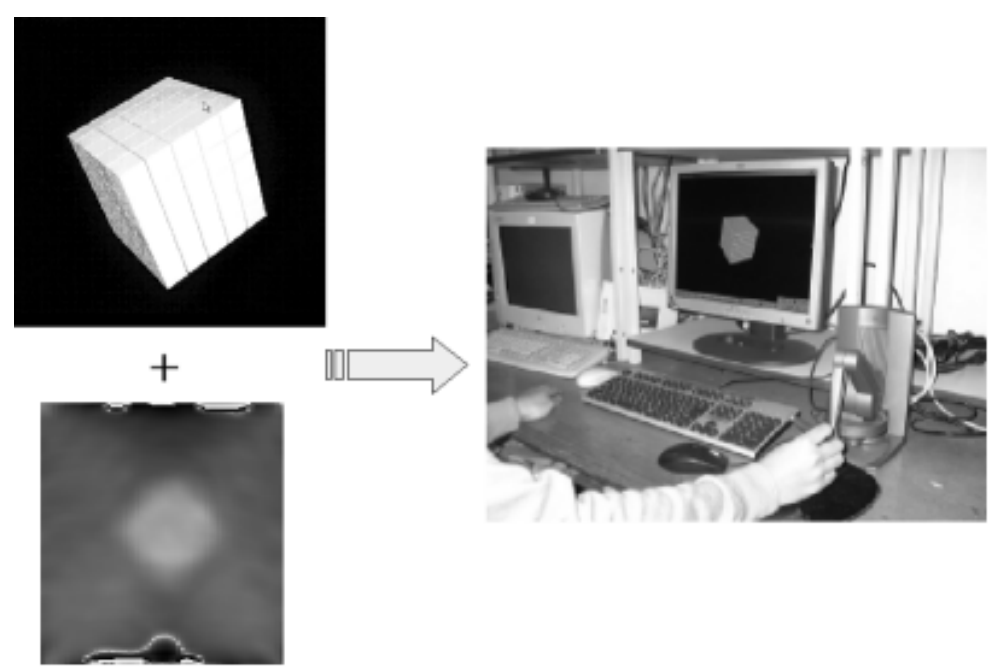

Figure 4: Virtual palpation system.

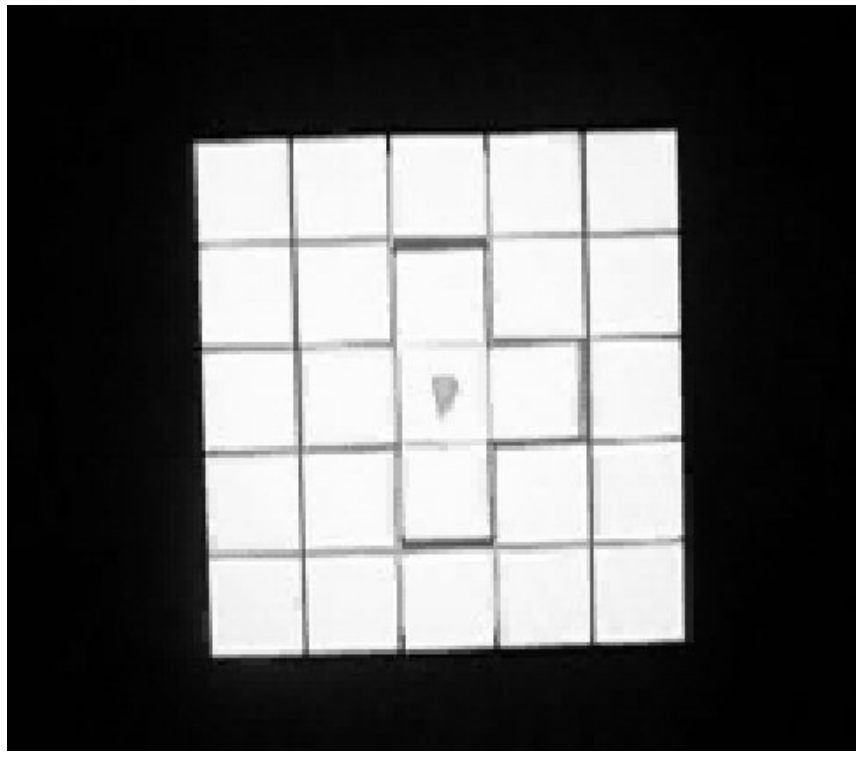

Figure 5: Representation of Human Haptics. 


\subsection{Representation of Human Haptics}

Figure 5 shows a representation of human haptics. Not only the pressed cube but four cubes around the pressed cube move in the direction of the pressure. This movement corresponds to a dent of the surface of the body at palpation. Haptic sense (force feed-back sense) also can be felt by finger via PHANToM Desktop $^{\mathrm{TM}}$ simultaneously. The movement and haptic sense are determined by the physician or medical doctor based on elasticity information and the mass of the cubes subjectively and empirically. This determination is finished by a user, based on the user's sense in advance to practical use. Therefore, the user's own Human haptics can be calculated and presented in real time for practical use.

In Figure 5, the arrow cursor means PHANToM Desktop ${ }^{\text {TM }}$ cursor. When PHANToM Desktop ${ }^{\text {TM }}$ is manipulated in real space, the cursor moves according to movement of PHANToM in 3D virtual space and feeds a reaction force back to the finger. The user can feel different haptic sense caused by the difference of the depth of tumor.

\section{Conclusions}

Recently, medical ultrasonic elasticity imaging technique has been developed. It is expected to realize a "virtual palpation system" for accurate comprehension of the elasticity information by a medical doctor and ultrasonographer.

In this research, a typical haptic device, PHANToM Desktop ${ }^{\mathrm{TM}}$ is applied to ultrasonic elasticity imaging to realize a virtual palpation system. Furthermore, to accomplish real-time representation of Human Haptics, reduction of computational cost is attempted. In this proposal, a target tissue is expressed by many small rigid cubes in 3D virtual space; movement of cubes, which means a dent of the surface of the body at palpation, is calculated by using the mass and elasticity information of each cube (Spring-damper model) and haptic sense, in the other words, force feed back, is also determined by the physician or medical doctor based on mass and elasticity of each cube subjectively and empirically. In this proposal, this determination is finished by a user, based on the user's sense, in advance to practical use. The determined data is saved in the system as the user's own calibration data. Therefore, the constructed system enables each user to feel own Human Haptics in real time. The typical technique for representation of Human Haptics, which is the Finite Element Method, is not used in this proposal since its computational cost is significantly high.

Future works are how put the human haptics close to real haptic sense quantitatively and an application of this system to a practical diagnostic system.

\section{Acknowledgment}

This work was supported in part by the Ministry of Education, Culture, Sports, Science and Technology, Japan, under a Grand Aid for Scientific Research B(2) (No.15300284). 


\section{References}

[1] Bamber, J.C., Barbone, P.E., Bush N.L., Cosgrove, D.O., Doyely, M.M., Fuechsel, F.G., Meaney, P.M., Miller, N.R., Shiina, T. \& Tranquart, F., Progress in Freehand Elastography of the Breast. IEICE Trans. Inf. \& Syst., Vol.E85-D, No.1, pp.5-14, 2002.

[2] Suzuki, N., Hattori, A., Takatsu, A., Uchiyama, A., Kumano, T., Ikemoto, A., \& Adachi, Y., Virtual surgery simulator with force feedback function. Proceedings of the $20^{\text {th }}$ Annual International Conference of the IEEE Engineering in Medicine and Biology Society, Vol.20, No.3, pp.1260-1262, 1998.

[3] Nakao, M., Komori, M., Oyama, H., Matsuda, T., Sakaguchi, G., Komeda, M. \& Takahashi, T., Haptic Reproduction and Interactive Visualization of a Beating Heart Based on Cardiac Morphology. 2001 International Medical Informatics Association, MEDINFO 2001, eds. Patel, V., et al., IOS Press. Amsterdam, pp.924-928, 2001.

[4] Sarama, S., Virtual Haptics. Proceedings of the 9th IVR seminar, pp.75-104, 2001. 